\title{
A co-located collaborative Augmented Reality application
}

\author{
Susanna Nilsson* \\ Dept. of Computer and Information Science \\ Linköping University, Sweden
}

\author{
Björn J.E. Johansson \\ FOI \\ Sweden
}

\author{
Arne Jönsson \\ Dept. of Computer and Information Science \\ Linköping University, Sweden
}

\begin{abstract}
This paper presents results from a study on using an AR application to support collaborative command and control activities requiring the collaboration of three different civil service organisations. The technology is used to create a common ground between the organisations and allows the users to interact, plan resources and react to the ongoing events on a digital map. The AR application was developed and evaluated in a study where a forest fire scenario was simulated. Participants from the involved organisations acted as command and control teams in the simulated scenario and both quantitative and qualitative results were obtained. The results show that AR can become a useful tool in these situations in the future.
\end{abstract}

\section{Introduction}

In complex collaborative situations, such as command and control in crisis management, personnel from different domains and organisations often must work together [Cross and Bopping 1998]. Commanders from various organisations must set goals and coordinate action under time pressure. However, collaborative work across organisational borders is not simple and confusion emerging from differences in terminology is not rare. Information presented to the participants of the collaborative task has to be simple enough to support cooperation between people from different organisations but at the same time be rich enough for an individual from a specific organisation to facilitate her decision making. We believe that Augmented Reality (AR) has potential to support collaboration between personnel from different organisations. AR is based on the concept of presenting virtual information in the perceptual field of a user, thus allowing the user to interact with virtual information as well as her real physical surroundings in a non-intrusive way. AR further allows for both independence and individuality [Billinghurst and Kato 2002] meaning that each person can independently have data tailored and presented according to her needs in various situations. AR also supports cooperation [Billinghurst and Kato 2002] as the users can cooperate in a natural way while seeing and interacting with both their own and each other's data.

Our primary research question in this paper is whether users experience that the AR tool facilitates cooperation and helps them in establishing and maintaining a shared situational picture when working jointly to solve a dynamic decision making task. The aim is partly to describe the system design and evaluation process but the main focus of the paper is the results generated by the end user study concerning the AR systems' ability to support collaboration and shaping of common ground between the participants.

\footnotetext{
*susni@ida.liu.se

†bjorn.j.e.johansson@foi.se

‡arnjo@ida.liu.se
}

Copyright $(2009$ by the Association for Computing Machinery, Inc.

Permission to make digital or hard copies of part or all of this work for personal or classroom use is granted without fee provided that copies are not made or distributed for commercial advantage and that copies bear this notice and the full citation on the first page. Copyrights for components of this work owned by others than ACM must be honored. Abstracting with credit is permitted. To copy otherwise, to republish, to post on servers, or to redistribute to lists, requires prior specific permission and/or a fee. Request permissions from Permissions Dept, ACM Inc., fax +1 (212) 869-0481 or e-mail permissions@acm.org.

VRCAI 2009, Yokohama, Japan, December 14 - 15, 2009.

(c) 2009 ACM 978-1-60558-912-1/09/0012 $\$ 10.00$

\section{AR to support collaborative work}

There are evaluations of using AR to support collaborative work compared to how similar work is conducted without AR [Kiyokawa et al. 2002; Billinghurst et al. 2002]. What they found was that their system did in fact exceed the expected outcome and that AR is a promising tool for collaborative work. However, few, if any, AR systems have actually been aimed for use in crisis or emergency management situations which are examples of real world situations where collaboration is essential. Emergency management often demand collaboration between different organisations, not least at the command and control level.

In many system designs, it is often assumed that merely providing a shared representation would be enough to facilitate a shared understanding of a situation when a team of decision makers work together. However, linguists and psychologists have observed that in reality, meaning is often negotiated or constructed jointly [Clark 1996]. Although providing the same view of a situation to two or more people is a good starting point for a shared understanding, things like professional and cultural background, as well as expectations formed by beliefs about the current situation, clearly shape the individual interpretation of a situation. Clark [1996] denotes the knowledge two or more individuals have when entering a joint activity 'common ground'. Common ground is the least shared understanding of the activity that the participants need to have in order to engage in a joint action with a higher goal than creating common ground. The maintaining of common ground is an ongoing process, which demands both attention and coordination between the participants. Exercising command and control is an attempt to establish common intent to achieve coordinated action [McCann and Pigeau 2000]. Successful communication is necessary to achieve this.

There are also situation specific problems that emerge in collaborative command and control tasks. Such tasks often circle around a shared representation of the current activities, as in the case of a situational map. Most organisations involved in command and control tasks, like the military or rescue services, have developed a library of symbols that can be utilised for representing units and events. A problem arises when representatives from different organisations work together, since they are used to working with their own organisation-specific symbols and conventions. This means that time has to be spent on explaining and negotiating meaning when jointly creating and manipulating a shared representation. This can be a tedious task to undertake when there is little time, as for example in the case of forest fire-fighting in, or close to, urban areas. Thus, providing means to facilitate establishing a common ground is important for efficient collaboration. Furthermore, for each organisation there is information that is only interesting for the representatives from that organisation. From this perspective, commanders from different organisations need personalised views of the same situational map. AR has the potential to provide both of these aspects and in doing so it may improve initial common ground.

\section{Method}

Introducing new technology in a specific domain affects not only the user, but also the entire context of the user, and most notice- 
ably the task that the user performs. In addition to this, the new design, or tool, will change the task, rendering the first analysis invalid [Hollnagel and Woods 2005; Woods and Roth 1988]. Consequently, studying usefulness of technology in isolation from the natural context (as in many traditional, controlled usability studies) may not actually reveal how the technology will be used and accepted in reality. Understanding, and foreseeing the effects of change on both user, task and context requires knowledge about the system, but perhaps even more importantly, an understanding of the context, user and user needs [Hollnagel and Woods 1983].

The approach to naturalistic studies of human-machine interaction adopted in this study is called Cognitive Systems Engineering (CSE) [Hollnagel and Woods 1983; Hollnagel and Woods 2005]. The main idea in the CSE approach is the concept of cognitive systems, where the humans are a part of the system, and not only users of that system. The focus is not on the parts and how they are structured and put together, but rather on the purpose, and the function of the parts in relation to the whole. This means that rather than isolating and studying specific aspects of a system by conducting laboratory studies, or experiments under controlled conditions, users and systems should be studied in their natural setting, doing what they normally do.

As a result of this theoretical approach, the study included a predesign phase where field experts from three different organisations (fire and rescue services, police department and the helicopter platoon in the local area) took part in a brainstorming session to establish the parameters of the AR system. This brainstorming session was used to define the components of the software interface, such as what type of symbols to use, and what type of information is important and relevant in the task of creating common ground between the three participating organisations. Based on an analysis of the brainstorming session a first design was implemented. This design was evaluated using a scenario in which the participants, one from each of the three organisations, had to interact and work together as they would in a real situation, cooperating in response to a forest fire. The exercise was observed and the participants also answered questionnaires pertaining to the AR system design, and finally a focus group discussion was held. As a result of this design evaluation the system was redesigned and this redesign was later evaluated through a second study consisting of a focus group discussion and observation of the users interacting with the redesigned system while performing simple tasks. The final outcome of this design process is the system and scenario described in this paper.

\subsection{Participants}

In order to find real world applicable results we need real world end users. To meet this demand participants from three different organisations involved in crisis management were recruited. In total 30 participants took part of the study during ten sessions distributed over ten days, with three participants in each session. The participants were all at the level in their organisation where they in real life are assigned to team-coordinating situations. This means that they all either have experience from working in teams with partners from at least one of the other organisations, or have a position in their organisation which require that they have a set level of education and training in these types of command and control assignments. The groups formed here had never worked together before and they did not know each other prior to this study. Of the ten trials, two were spoiled due to unforeseeable events (in one case one participant was called to active duty due to an emergency and in the other case external technical problems forced the trial to end prematurely). This resulted in a total of eight complete trials with 24 participants, of which 23 were male, one female and the ages ranged from 25 to 57 (median: 36, average: 39,1). The gender inbalance reflects the

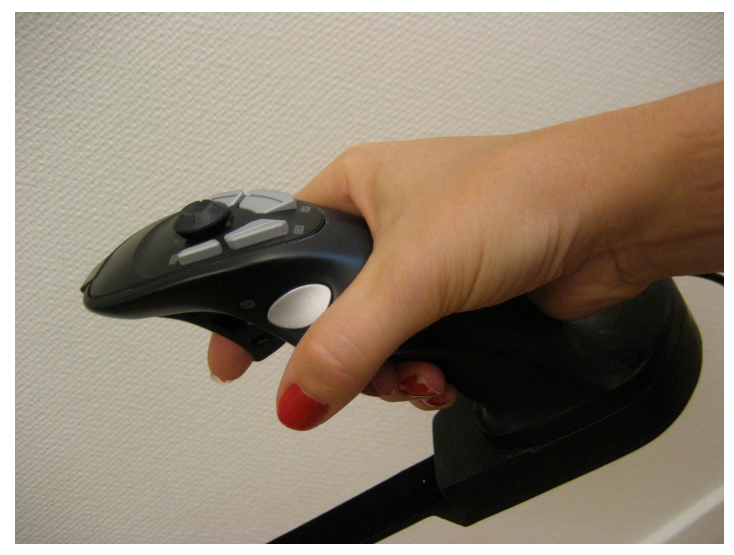

Figure 1: Joystick interaction device

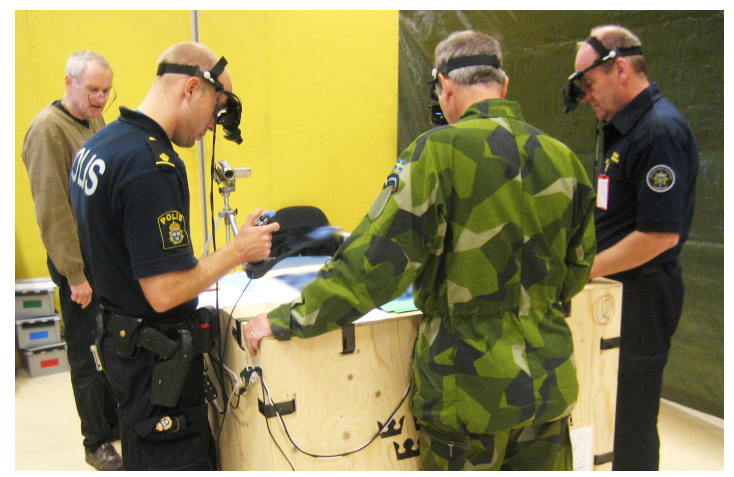

Figure 2: The simulated natural setting (a helicopter base)

gender distribution of the user groups.

\subsection{Task scenario and procedure}

It is not possible to conduct experiments in a real life fire-fighting situation. Instead, to create a realistic study, we used a scenario where the groups had to collaborate, distribute resources and plan actions in response to a simulated forest fire and other related or non-related events. In the scenario the participants act as they would as on-scene commanders in their respective organisation. This means that they together have to discuss the current situation and decide how to proceed in order to manage the fire, evacuate residents, redirect traffic, coordinate personnel as well as dealing with any unexpected events that may occur during such incidents. Normally discussions like this take place in a temporary control room (often a room at an appropriate location near the affected area) around a paper map of the affected area. The AR system was used as a tool for them to see and manipulate their resources on a digital map and as a way to have an overall view of the situation.

In the study the participants collaborated in groups of three, with one commander from each organisation (the fire and rescue services, the police department and the helicopter platoon) in every team. The participants had to interact and work together to complete assignments in a dynamic scenario. The team worked together around the same table to encourage face-to-face communication as this is an important part of collaborative planning processes.

The study was conducted at a helicopter base, Figure 2. In order to create a dynamic scenario and realistic responses and reactions to the participants' decisions in the three sessions, we used a gam- 
ing simulator, $C^{3}$ Fire [Granlund 2001]. $C^{3}$ Fire generates a task environment where a simulated forest fire evolves over time. The simulation includes houses, different kinds of vegetation, computer simulated agents, vehicles etc. that can be controlled by an experiment assistant. The simulator was run in the background by the research team where one member inserted information into the gaming simulator, for instance, that several police cars have been reallocated to attend to a traffic incident. The experiment manager acted as a feedback channel to the participants in order for them to carry out their work. For instance, when the reallocated police cars had reached their new destination the experiment leader returned with information to the participants.Other examples of information from the gaming simulator are weather reports, status and location of personnel and vehicles, the spread of the fire etc.

Three different scenarios were used, each describing a forest fire that had been going on for a couple of days. The description was rather detailed and included information on when the fire had started, where people had been seen, weather conditions etc. Each organisation had a number of units that they had to assign to different locations on the map as they would have done in a real situation. They can independently place symbols using the handheld interaction device and they can also discuss with each other how to place their own symbols and also shared symbols, such as the fire symbol and break points.

To create a baseline for the evaluation the scenario was also conducted using paper maps and pens (similar to how they would normally go about this type of task). Every team performed the exercise over a course of about three hours where time was spent accordingly: 30 minutes introduction to the project and procedure, 30 minutes of training where the participants did a test run of the procedure both using the paper map and using the AR system, and then three 20 minute sessions where the forest fire scenario was played out with a number of different events in every session.

In the first session the participants worked with the AR system, in the second session they worked with a paper map, pens and transparency film, and in the third session they used the AR system again. After each of the three sessions the participants filled out a questionnaire regarding the session and after the final session they also filled out an additional questionnaire which focused on an overall evaluation of the AR system. The questionnaires used six-point Likert items and also had open-ended questions. Finally a focus group discussion was held where the participants could reflect on and discuss a series of topics relating to the exercises, the scenario and the AR system.

\subsection{The Augmented Reality System used in the study}

A multi user collaborative AR application was designed, evaluated and redesigned based on the results from evaluation with real users [Nilsson et al. 2008]. The AR system used in the study was a high fidelity prototype, which allowed the users to interact with virtual elements. It includes a Z800 3DVisor from eMagin (http: //www.3dvisor.com/) integrated with a firewire camera. The Mixed Reality system runs on a $2.10 \mathrm{GHz}$ laptop with 3 GB RAM and a 128 MB NVIDIA GeForce 8400M GS graphics card and the marker tracking software used is based on ARToolkit [Kato and Billinghurst 1999]. In order to interact with the AR system the users had a joystick-like interaction device allowing them to choose objects and functions affecting their view of the digital map, see Figure 1.

One important technical design feature, which was a result of the iterative design process, is the ability to point in the map. The system allows the users hand to be superimposed over the digital map image, see Figure 3.

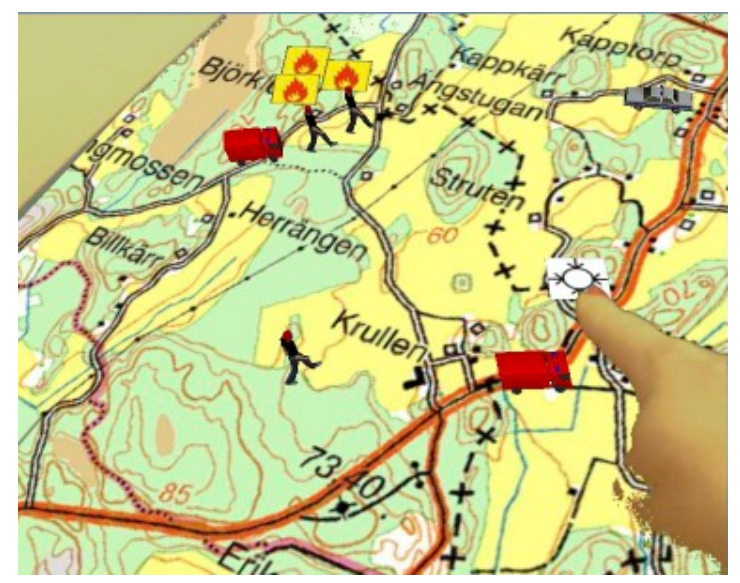

Figure 3: The users display showing the digital map with symbols and pointing used in the collaborative AR application

The users have access to a personal, organisation-specific symbol library which they can use to create a situational picture. Examples of symbols are police vehicles, fire trucks, helicopters, and personnel. Other types of symbols are the function symbols, for instance the (1) symbol which when used allows the user to see additional organisation-specific information about the already placed symbols, such as information about how many hours personnel has been on duty, or how much water is left in the tank of a tank truck. The symbols are simplified to some degree in order to be understandable by users from other organisations. All symbols are three-dimensional and follows the users' movements, e.g. if a user kneels down the symbols are seen from the side. It is also possible to personalise the system by filtering out symbols belonging to one or more organisation, thus, e.g. showing only symbols from the own organisation on the map.

If necessary, the users can manipulate each other's symbols, e.g. a fire-fighter can place, delete and move a police vehicle. There are also a set of symbols that are common to all users of the systems, such as fires and smoke (this is particularly important in this case as the participants in the study are confronted with a forest-fire fighting task). The users thus have access to a digital 'playground' where they can add symbols, move them or remove them freely. The symbols were placed in relation to a marker attached on a joystick, meaning that there was no fixed menu in the user's field of view or related to the map. Instead the menu of symbols was related to the physical controller.

Users can use a command function to zoom in and out on the map to focus on a symbol or a part of the map. It is also possible to physically lean in over the map to get a closer look, as you would over a regular paper map. In order to place a symbol the user first moves the joystick-attached marker to the chosen position on the map and then selects and places the symbol in the menu by using the buttons on the joystick. The same procedure is used to remove a symbol, to see additional information about a symbol or to zoom in the map.

\section{Results}

The AR-system collaboration questionnaire comprised 15 items, and 6 open-ended questions. The queries and data from the sessions are presented in Figure $4^{1}$. The open-ended responses are presented and discussed in the following text.

\footnotetext{
${ }^{1}$ The queries are translated to English by the authors.
} 


\begin{tabular}{|c|c|c|c|c|c|c|}
\hline & \multicolumn{2}{|c|}{ AR1 } & \multicolumn{2}{|c|}{ AR2 } & \multicolumn{2}{|c|}{ Paper } \\
\hline Request item & $\mu$ & $\sigma$ & $\mu$ & $\sigma$ & $\mu$ & $\sigma$ \\
\hline 1. It took a long time to start to co-operate & 4.083 & 1.380 & 5.333 & 0,963 & 5.333 & 1.090 \\
\hline 2. It was easy to co-operate & 4.739 & 0.964 & 4.696 & 1.550 & 5.087 & 1.474 \\
\hline 3. I think that AR-systems are good tools to use for co-operation & 4.333 & 1.050 & 4.417 & 1.136 & 4.250 & 1.391 \\
\hline 4. The co-operation was fun & 5.250 & 0.794 & 5.292 & 0.690 & 5.208 & 0.833 \\
\hline 5. I felt that the group controlled the situation & 4.167 & 1.090 & 4.792 & 1.141 & 5.000 & 0.885 \\
\hline 6. It was easy to mediate information between the organisations & 4.042 & 0.859 & 4.833 & 0.917 & 5.0 & 0.834 \\
\hline 7. The map made it easy to achieve a common situational picture & 5.125 & 0.076 & 5.041 & 0.999 & 4.167 & 1.404 \\
\hline 8. The symbols made it easy to achieve a common situational picture & 5.000 & 1.063 & 4.833 & 1.050 & 3.417 & 1.472 \\
\hline 9. The map became cluttered/messy & 3.708 & 1.488 & 4.000 & 1.474 & 2.375 & 1.469 \\
\hline 10. I would have liked to have had more information than what was available & 2.750 & 1.422 & 3.292 & 1.459 & 3.167 & 1.659 \\
\hline 11. I felt that I was certain that I could interpret what was on the map & 3.708 & 1.488 & 4.542 & 1.132 & 3.750 & 1.452 \\
\hline 12. The map helped me trust the situational picture & 4.042 & 1.233 & 4.667 & 1.090 & 3.667 & 1.373 \\
\hline 13. The symbols helped me trust the situational picture & 3.958 & 1.268 & 4.582 & 1.060 & 3.500 & 1.474 \\
\hline 14. I though I had a good situational picture & 4.083 & 1.140 & 4.542 & 1.103 & 4.250 & 1.032 \\
\hline 15. I thought the others had a good situational picture & 4.417 & 0.881 & 4.500 & 1.142 & 4.458 & 0.977 \\
\hline
\end{tabular}

Figure 4: AR-system questionnaire, average score and standard deviation. As the statements in the questionnaire were both positively and negatively loaded (see for instance the first two items), the scores on the negatively loaded items were transformed in order to make the result easier to interpret. This means that in the table a high score is positive for the AR system/paper map and a low score is negative for the AR system/paper map.

In general the results on the questionnaire were positive for the AR system. The average scores were all above 3 out of 6 which is relatively good for a new system. Using one way ANOVA with Bonferroni post hoc tests we found significant differences between the three session on items $1,5,6,7,8,9,12$ and 13 .

There is a significant difference between the first AR session (AR1) and the second AR session (AR2) on the first item, Item 1, regarding how long it took to begin working together. The participants felt that it took longer time to cooperate in the first AR session, see Figure 5, left. In AR2 they felt that they began to collaborate as fast, or faster, as when they used the paper map $(\mathrm{F}(2,42)=12,8, \mathrm{p}<0.05)$.
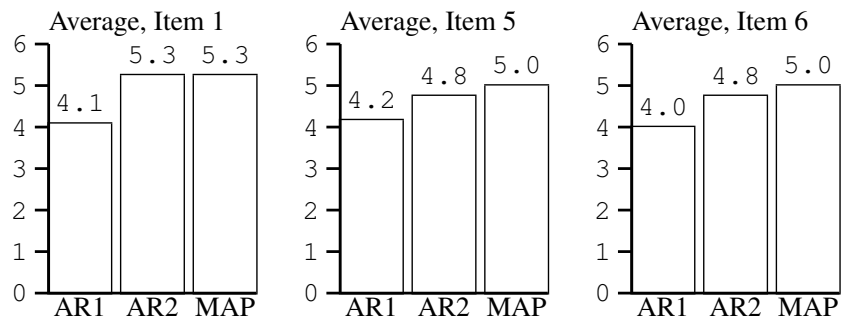

Figure 5: Results from items 1 (It took a long time to start to cooperate), 5 (I felt that the group controlled the situation) and 6 (It was easy to mediate information between the organisations). For further explanation, see text.

As one user commented:

"Since we were a bit used to it, we could use the technology in a better and more effective way". (RS0924, Question 3:4) ${ }^{2}$

When asked if it was easy to collaborate, Item 2, the results were positive in all three sessions - the mean score was $4.7,4.7$ and 5.0 on a 6 grade scale. There was in this case a slight difference between the organisations, where the rescue services scored lower than the

\footnotetext{
${ }^{2}$ In the following text quotes of the participants are coded as follows: the first letter/s indicate organisation (P-Police, RS- Rescue services, HPHelicopter Pilot), the following four numbers are the team number, and the final number combination indicates which of the open-ended questions the quote is related to.
}

helicopter pilots $(\mathrm{F}(2,42)=2.8, \mathrm{p}<0.05)$. Although there were no significant effects bewteen the first and second AR session there is a social effect of getting to know one another better and therefore being able to understand and collaborate better:

"It worked smoothly with suggestions and orders. Mainly because of the shared picture and also since we are beginning to find our feet". (HP0926, Q3:4)

When asked about the AR system as a tool for collaboration, Item 3 , again the scores were high. There was no significant difference between the sessions. There were however some differences between the organisations, where the helicopter pilots appreciated the AR system slightly more than the rescue service participants $(\mathrm{F}(4,42)=5.1, \mathrm{p}<0.05)$.

Concerning whether or not the participants enjoyed the collaboration, Item 4, the scores are high, between 5.2 and 5.3. There was no significant difference between the sessions, see Figure 4, all seemed to enjoy it and the means were 4.3 at the lowest.

On the question of feeling that the group had control over the situation, Item 5 , we note the importance of training. We have a significantly lower value for the first AR session, (Figure 5, middle) indicating that users have the same sense of control using the AR system as they have using a normal paper based map, after some training. In AR1 the average score was 4,2 while the average in the second AR session was $4.8(\mathrm{~F}(2,42)=7.98, \mathrm{p}<0.05)$. In the paper session the average was 5.0 and this was also significantly higher than in AR1 $(\mathrm{F} 2(42)=7.98, \mathrm{p}<0.05)$. There was no significant difference between the paper map session and AR2.

Another aspect of collaboration is sharing information, Item 6, and this activity was more difficult during the first AR session. The overall average score on the item regarding information sharing, Item 6, was high; 4.0 out of 6 in AR1 and 4.8 in AR2 and 5,0 in the paper session, see Figure 5, right. The difference was significant between AR1 and AR2 $(\mathrm{F}(2,42=12.0, \mathrm{p}<0.05)$ and between AR1 and the paper map $(\mathrm{F}(2,42)=12.0, \mathrm{p}<0.05)$. However, there was no significant difference between the second AR session and the paper map session which may indicate that sharing information was experienced as easy to do while working on the paper map as with the AR system after some training.

A group of items specifically addressed the map and the symbols on the map; Item 7, Item 8 and Item 9. Here the scores for 
Average, Item 7

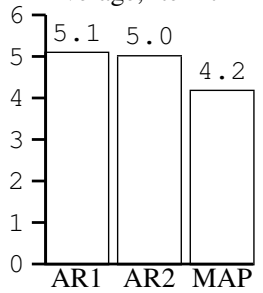

Average, Item 8
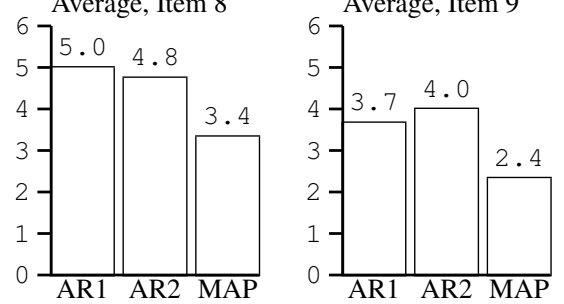

Figure 6: Results from items 7 (The map made it easy to achieve a common situational picture) , 8 (The symbols made it easy to achieve a common situational picture) and 9 (The map became cluttered/messy). See text for further explanation.

the AR system are higher than for the paper map, Figure 6, suggesting that the use of the AR system made it easier to achieve a common situational picture. Regarding the map, Item 7, we only see a tendency to difference between AR2 and the paper map $(\mathrm{F}(2,42)=6.1, \mathrm{p} \approx 0.052)$, but regarding the symbols, Item 8 , there is a significant difference. The symbols in AR2 made it easier to achieve a common situational picture compared to the paper map $(\mathrm{F}(2,42)=15.3, \mathrm{p}<0.05)$. The map is also regarded as less messy when using the AR system, Item 9, with significant differences both the first and second time the AR system was used, AR1 vs paper map $(\mathrm{F}(2,42)=12.7, \mathrm{p}<0.05)$ and AR2 vs paper map $(\mathrm{F}(2,42)=12.7$, $\mathrm{p}<0.05)$.

We also note that the users wanted even more symbols than we had on the map, Item 10, scoring rather low on this item in all three situations. The participants had, however, no problems to interpret the symbols, Item 11 . When asked if the map and symbols helped the participants trust the situational picture, Item 12 and Item 13, there are differences. Concerning whether the map helped the users trust the situational picture, Item 12, we have a tendency to difference between the paper map and the second usage of the AR system, $\mathrm{AR} 2$, on the map, Item $12(\mathrm{~F}(2,42)=4.6, \mathrm{p} \approx 0.051)$. The symbols, Item 13, helped the users more using the AR system, AR2, than the symbols on the paper map $(F(2,42)=5.1, p<0.05)$. We also found a significant difference between the first and second use of the AR system, AR1 vs AR2 for Item $12(\mathrm{~F}(2,42)=4.6, \mathrm{p}<0.05)$ and for Item $13(\mathrm{~F}(2,42)=5.1, \mathrm{p}<0.05)$.
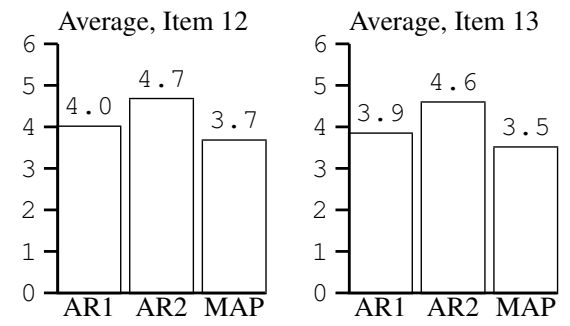

Figure 7: Results from items 12 (The map helped me trust the situational picture) and 13 (The symbols helped me trust the situational picture). See text for further explanation.

Finally we had two items, Item 14 and Item 15, where users had to provide a more subjective view of the situational picture. Our participants scored high on these items in all three situations, all above 4 , but there were no significant differences between the sessions or organisations.

\section{Discussion}

On many items the participants scored the system higher in the second session with AR (AR2) as compared to the first session (AR1).
This indicates the necessity of more than one trial or session with the AR system. This is probably valid in most studies examining new technologies. If the study had been designed with only one AR session (apart from the training session) the results would have been less positive for the system. This would not have been a fair comparison towards the baseline session as the participants are all familiar with paper maps but have never before encountered a system like the one in this study. Another aspect of several sessions is the social effect of collaborative work. As several participants pointed out in the questionnaire, it became easier to both use the system and communicate with each other in the second AR session. This is partly due to the training effect on the AR system, but also due to the fact that the particpants got to know each other better.

The participants experienced that it was simple to understand the symbols provided, although they requested to have even more symbols to choose from. Adding symbols, and information to symbols, is a simple issue to solve in the AR system. Again, our focus in this study was not to evaluate a complete system, but the prospects of using AR for cooperation.

The information sharing aspect of the system turned out to be equivalent in the AR system and the paper map which is a very promising result. The current technical solution, using a camera rather than a see-through display, causes a lack of direct eye contact which could be a drawback as gazing is used frequently as an indicator of focus in face-to-face communication. Despite the lack of eye contact the participants felt that they could easily share information among each other. This could be explained by the AR system's ability to present a common situational picture when everyone sees what everybody else does with their resources directly. This reduces the need to actively request and present information as part of the information sharing process:

"Good! It gives a credible situational picture and when you are secure in using the system my hope is that you can focus more on your task and less on verbal communication" (RS0924, Q3:6)

The ability to see each other's units may also have strengthened the perception of them being a team rather than just participants of their respective organisations:

"I felt that we could cooperate in a good way with this technology since we could see each others units. It became one operation together instead of like it is today when we work in different places although it is the same event." (P0930, Q 2:4)

The difference between the organisations regarding the AR systems potential as a collaborative tool may perhaps be explained by the different responsibilities and experiences of the organisations in operations like the one in the scenario. While the police participants in the focus group discussion commented on the lack and need of new technology in their organisation it seemed to be the opposite for the other two organisations. The rescue services had just recently installed new systems in their control room environment and the helicopter pilots are currently going through a process of changing the current helicopter technology into new. This could explain why the participants from the police department were more positive towards adding new technology to their workplace than the other participants.

"An instrument that would work in reality/real life" (P0923, Q1:6)

There are of course problematic issues with the system, and primarily these problems were related to interaction aspects rather than the content of the information: 
"Takes too much time to move the resources" (RS0923, Q1:6)

This was a drawback in the design - the tested system was a prototypical system and not all design choices had been evaluated. In order to have openness in the system all organisations could see all available symbols in the menu, which means that they also needed to go through them while changing the resources and contents of the map. This was not experienced as a major problem in the pre study trial and was hence neglected in the design process. Clearly this is an aspect of the system that needs to be addressed in future development. However, the participants did not consider any of the symbols unnecessary, but in further development the interaction will be re-designed. Personalising the menu and symbol selection will make the interaction even easier and less time consuming.

However, the positive aspects of the new technology seemed to outweigh the negative in most instances, and this quote can perhaps illustrate why:

\section{"So many advantages to have a complete overview"} (P0924, Q1:6)

In emergency management and collaborative command and control operations the general overview of the situation is important for the team to achieve a common picture of the ongoing event. Having a complete overview of available resources and where they are located is invaluable for information sharing and decision making. The real time overview given by the AR system is a major contribution to the creation of a common ground for the collaborative work:

\begin{abstract}
"It was easy and clear to see the others units. Good that you can point on the map with your hand and in that way show where you mean, good that you see the others point in order to help each other out. That you are in the same room, with the same map simplifies tremendously." (HP0930, Q 1:4)
\end{abstract}

\section{Conclusions and future direction}

We have in this paper presented a co-located collaborative AR application which enables the users to share information and actively take part in an ongoing scenario. The aim of this study was not to evaluate performance, but rather to evaluate the potential of the AR system in supporting the creation of a common situational picture in a collaborative team task and the user experience of the AR system. Neither the design of the study nor the scenario used allowed us to construct objective performance measures in a meaningful way. The analysis in this paper was therefore focused on the collaborative aspects of AR. We did not expect the AR system to gain higher acceptance than the paper map from the users, since paper maps and pens are part of the participants normal tools, while the AR system was a completely new experience for them. In general the results indicate that users are positive towards the system, and that they experience it as a support in building and maintaining a shared situational view. The system is thus a good candidate for a future collaborative support system for helping teams create common ground while coping with dynamic tasks.

The particular problem of enabling a shared view of gestures at the same time as presenting a virtual background was perhaps a consequence of adapting single-user AR technology to a multi-user setting. The system now allows augmentation of not only the individual users view but it allows each user to affect and change their team members view of the ongoing situation, which is fundamental to the definition of a collaborative AR system.

In this study, we have only examined how the AR technology can be used in a co-located setting. A future research focus could be to examine how AR can be used to support commanders working with a shared representation in a distributed setting. As the system supports deictic gesturing and collaborative manipulation of symbols even when the users are located separately, this could provide an interesting possibility.

\section{Acknowledgements}

This research is funded by the Swedish Defence Materiel Administration (FMV). The AR system was developed in close cooperation with XM Reality AB. We are deeply indebted to all the participants in our studies who volunteered their time and expertise to these projects.

\section{References}

Billinghurst, M., And Kato, H. 2002. Collaborative augmented reality. Communications of the ACM 45, 7 (July), 64-70.

Billinghurst, M., Kato, H., Kiyokawa, K., Belcher, D., AND POUPYREV, I. 2002. Experiments with face-to-face collaborative AR interfaces. Virtual Reality 6, 3, 107-121.

Clark, H. H. 1996. Using Language. Cambridge University Press, Cambridge.

Cross, M., And Bopping, C. 1998. Collaborative planning processes in command and control. In Fourth International in Command and Control Research and Technology, DoD CCRP.

GRANLUND, R. 2001. Web-based micro-world simulation for emergency management training. Future Generation Computer Systems.

Hollnagel, E., And Woods, D. D. 1983. Cognitive systems engineering: New wine in new bottles. International Journal of Man-Machine Studies 18, 6, 583-600.

Hollnagel, E., And Woods, D. D. 2005. Joint Cognitive Systems: Foundations of Cognitive Systems Engineering. CRC Press, Boca Raton, FL.

KATO, H., And Billinghurst, M. 1999. Marker tracking and hmd calibration for a video-based augmented reality conferencing system. In Proceedings of the 2nd International Workshop on Augmented Reality (IWAR 99), San Francisco, USA.

Kiyokawa, K., Billinghurst, M., Hayes, S., Gupta, A., SANNOHE, Y., AND KATO, H. 2002. Communication behaviors of co-located users in collaborative AR interfaces. In ISMAR, IEEE Computer Society, 139-148.

McCann, C., And Pigeau, R. 2000. The human in command. In The Human in Command; Exploring the Modern Military Experience, M. C and P. R, Eds. Kluwer Academic/Plenum Publishers; New York.

Nilsson, S., Johansson, B., And Jönsson, A. 2008. Design of augmented reality for collaboration. In Proceedings of VRCAI 2008, Singapore.

Woods, D. D., And Roth, E. M. 1988. Cognitive engineering: human problem solving with tools. Hum. Factors 30, 4, 415430. 